\title{
Factors affecting vaccine handling and storage practices among immunization service providers in Ibadan, Oyo State, Nigeria.
}

\author{
David M. Dairo, Oyarebu E. Osizimete
}

Department of Epidemiology and Medical Statistics, Faculty of Public Health, College of Medicine, University of Ibadan, Ibadan, Nigeria.

\begin{abstract}
Background: Improper handling has been identified as one of the major reasons for the decline in vaccine potency at the time of administration. Loss of potency becomes evident when immunised individuals contract the diseases the vaccines were meant to prevent.

Objective: Assessing the factors associated with vaccine handling and storage practices.

Methods: This was a cross-sectional study. Three-stage sampling was used to recruit 380 vaccine handlers from 273 health facilities from 11 Local Government areas in Ibadan. Data was analysed using SPSS version 16

Results: Seventy-three percent were aware of vaccine handling and storage guidelines with $68.4 \%$ having ever read such guidelines. Only $15.3 \%$ read a guideline less than 1 month prior to the study. About $65.0 \%$ had received training on vaccine management. Incorrect handling practices reported included storing injections with vaccines $(13.7 \%)$ and maintaining vaccine temperature using ice blocks (7.6\%). About $43.0 \%$ had good knowledge of vaccine management, while $66.1 \%$ had good vaccine management practices. Respondents who had good knowledge of vaccine handling and storage [OR=10.0, 95\%CI $(5.28-$ 18.94), $\mathrm{p}<0.001]$ and had received formal training on vaccine management [OR=5.3, 95\%CI $(2.50-11.14), \mathrm{p}<0.001]$ were more likely to have good vaccine handling and storage practices.

Conclusion: Regular training is recommended to enhance vaccine handling and storage practices.

Keywords: Vaccine handling, storage practices, immunization, service providers, Ibadan, Nigeria.

DOI: http://dx.doi.org/10.4314/ahs.v16i2.27

Cite as: Dairo DM, Osizimete OE. Factors affecting vaccine handling and storage practices among immunization service providers in Ibadan, Oyo State, Nigeria. Afri Health Sci 2016;16(2): 576-583. http://dx.doi.org/10.4314/ahs.v16i2.27
\end{abstract}

\section{Introduction}

The development of vaccines for the prevention of infectious diseases is one of the most significant accomplishments of public health in the last century. The efficacy of a vaccine in preventing disease depends largely on the quality of the immunizing agent. Failure to adhere strictly to recommended specifications for vaccine han-
Corresponding author:
David M. Dairo,
Department of Epidemiology and
Medical Statistics, Faculty of Public
Health, College of Medicine, University
of Ibadan, Ibadan, Nigeria.
E-mail: drdairo@yahoo.com
Tel: +2348035664708

dling and storage can render vaccines useless. Inactivation of a vaccine may become evident only after immunised individuals acquire the disease the vaccine was designed to prevent. The identified cases are often considered primary or secondary vaccine failures, thereby obscuring the role that improper vaccine handling may have played in the failure.

A study conducted in Lagos, showed that $3 / 4$ of the vaccines at the local government level were sub-potent with the situation failing to improve over the three-year study period $^{1}$. Studies have also reported that improper vaccine storage leading to the administration of sub-potent vaccines may have been associated with outbreaks of vaccine preventable diseases in Israel $^{2}$ and several developing countries $^{3}$.

Poor handling and storage, cold chain deficiencies such as inadequate infrastructure, power shortages and poor maintenance are associated with vaccine wastage. Though there are very few countries reporting vaccine wastage to 
World Health Organization, WHO (19 of the 72 Global Alliance for Vaccine Initiative, GAVI eligible countries), developing countries have been found to incur cost of vaccine wastage in excess of $\$ 4$ million and $\$ 6$ million for pentavalent and pneumococcus vaccines respectively. It is noteworthy that Nigeria is among the $74 \%$ of GAVI eligible countries that do not report vaccine wastage 4 .

Periodic assessment of vaccine handling and storage practices is instrumental in correcting wrong vaccine management practices and improving level of knowledge on vaccine management ${ }^{5,6}$. In addition, it identifies various capacity gaps (personnel and equipments), which when duly addressed strengthens the nation's immunisation service delivery, increases vaccination coverage and ensures the administration of potent vaccines to clients. This study was therefore conducted to assess the factors associated with vaccine handling and storage practices among immunization service providers in South-Western Nigeria.

\section{Methodology \\ Study area}

This study was conducted in Ibadan, the capital city of Oyo State, located in the SouthWestern part of Nigeria. The National Population Commission (NPC) 2009 estimate of the population of Ibadan puts it at 2.559 million ${ }^{7}$. There are 11 Local Government Areas (LGAs) for administrative purposes: five situated within the city and six surrounding LGAs. There are 289 Health Facilities conducting Routine Immunisation in Ibadan: 207 public and 82 private. The five LGAs within the city are catered for by 138 (89 public and 49 private) health facilities while the surrounding six LGAs have 151 (118 public and 33 private) health facilities. Antigens provided are BacileCalmette Guarin (BCG), Diptheria-Pertussis-Tetanus (DPT), Measles-Mumps-Rubella (MMR), Tetanus Toxoid (TT), Yellow Fever (YF), Poliomyelitis, Hepatitis B, and cerebrospinal meningitis vaccines. Immunization coverage for various parts of the city varies from full routine immunization coverage of $40.7 \%$ in the urban parts of the city and $37.9 \%$ in the rural areas of the city ${ }^{15,16}$.

\section{Sample size estimation}

The minimum sample size required for the study was derived from the Leslie Kish's formula for estimating single proportion:

$$
\frac{\mathrm{n}=\mathrm{Z} \alpha 2 \mathrm{pq}}{\mathrm{d} 2}
$$

where

$\mathrm{n}=$ minimum estimated sample size

$\mathrm{p}=0.578$ (proportion of vaccine handlers who recorded vaccine temperature twice daily). Set at $57.8 \%$ considering 'Cold Chain Status at Immunisation Centres in Ethiopia" by Berhane and Demissie, (2000).

$\mathrm{d}=$ degree of allowance of error set at 0.05

$\mathrm{n}=\frac{1.962 \times 57.8 \times 42.2}{0.052}$

$\mathrm{n}=$ minimum estimated sample size $=375$

assuming a non-response rate of $10 \%$,

$\mathrm{n}=\frac{\mathrm{N}}{1-0.1}=375 / 0.9 \quad=417$

\section{Sampling technique}

Three-staged sampling procedure was used to identify the respondents to be sampled.

Stage 1: Selection of LGAs: All 10 LGAs in Ibadan were selected to account for a large representative sample of health workers in Ibadan.

Stage 2: Selection of Health Facilities: The HFs were selected from each LGA using simple random sampling by balloting from a list of all HFs conducting RI.

Stage 3: Selection of respondents: Total sampling of all local immunisation and cold chain officers (at the local government storage sites), and all health workers (incharge of vaccine handling and storage) at health facilities was done.

\section{Eligibility criteria}

Inclusion criteria: Health workers who handle vaccines at health facilities; cold chain officers, and local immunisation officers who handle vaccines at the LGAs.

Exclusion criteria: Health workers who do not handle vaccines.

\section{Data collection procedure}

A validated semi-structured questionnaire, prepared in English language and translated to Yoruba language, with information on social demographic profiles of health workers, knowledge of vaccine handling and storage, and vaccine handling and storage practices, was used to interview health workers. All health workers fulfilling the inclusion criteria were interviewed at their places of work. 
Spot observation of the dry and wet vaccine storage conditions was carried out on the vaccine storage sites at LGA level using an observation checklist to assess vaccine storage conditions, minimum logistic and storage requirements, and storage and handling policies.

Knowledge of vaccine handling procedure was assessed on a 14 point scale containing questions on identity of each routine vaccine, appropriate storage conditions (temperature range), procedure for vaccine reconstitution, stages of changes in Vaccine vial monitors (VVM) and actions to be taken on vials with changed VVM. Respondents with scores of 10 and above $(75 \%)$ were categorised as having good knowledge.

Vaccine handling practice was assessed on a 12 point scale containing questions on respondents practice of storing other items together with vaccines in the refrigerators', frequency of temperature recording, information recorded on the vaccine and temperature logs, frequency of defrosting and alternate storage for vaccines during defrosting. Respondents with scores of 8 and above (75\%) were then categorized as having good practice .

\section{Statistical / data analysis}

Data entry and analysis was done using SPSS version 16.0 for windows. Respondents were described using frequencies, while bivariate analysis was used to identify factors associated with vaccine handling and storage practices. The dependent variable "vaccine handling and storage practices" was used for bivariate analysis. Variables found to be associated $(p<0.05)$ with vaccine handling and storage practices were entered into logistic regression model. These were awareness and utilization of vaccine handling and storage guideline, how recent a vaccine handling and storage guideline was utilized, formal training on vaccine handling and storage, and knowledge of vaccine handling and storage.

\section{Ethical considerations}

The research instruments were approved by the Oyo State Ministry of Health Ethical Review Board. Participants involved in the study were fully informed about the nature of the study, research objectives and confidentiality of the data. Thereafter their consent to administer the study instrument was requested. Participation in the interview was on voluntary basis. The names and any personal identifiers were not used in the survey questionnaires.

\section{Results}

\section{Socio-demographic characteristics of respondents}

A total of 380 health workers participated in the study. Mean age of respondents was $37.4 \pm 0.36$ years. Table 1 below shows the demographic characteristics of respondents. Respondents were mostly female $(77.6 \%)$ and a greater proportion $(65.3 \%)$ were less than 40 years of age. Eighty six percent were married as at the time of the study. Respondents (85\%) were highly educated, of Yoruba origin (96.3\%) and lived in urban areas $(70.0 \%)$

Table 1: Frequency distribution of respondents' socio-demographic characteristics

\begin{tabular}{|lll|}
\hline Characteristic & & Frequency (\%) \\
Age (in years) & $20-29$ & $39(10.3)$ \\
& $30-39$ & $209(55.0)$ \\
& $40-49$ & $89(23.4)$ \\
Gender & $50+$ & $31(8.2)$ \\
& Female & $295(77.6)$ \\
Ethnicity & Male & $85(22.4)$ \\
& Hausa & $1(0.3)$ \\
Religion & Igbo & $11(2.9)$ \\
& Yoruba & $366(96.3)$ \\
Marital status & Christianity & $245(64.5)$ \\
Residence & Islam & $118(31.1)$ \\
& Traditional & $3(0.8)$ \\
Highest level & Not Currently Married & $48(12.6)$ \\
education attained & Currently Married & $327(86.1)$ \\
& Urban & $266(70.0)$ \\
& Rural & $102(26.8)$ \\
& Secondary and below & $57(15.0)$ \\
& Post-Secondary & $323(85.0)$ \\
\hline
\end{tabular}




\section{Barriers to effective vaccine handling and storage}

Figure 1 shows challenges faced by respondents in vaccine handling and storage. Poor/unstable power supply $(86 \%)$ and lack of fuel for generators (64\%) were cited as the major challenges faced by vaccine handlers in storing vaccines. Others include insufficient/unavailable backup refrigerators $(52 \%)$, insufficient ice packs for cold temperature maintenance $(49 \%)$, high cost of transporting vaccines $(47 \%)$, insufficient cold boxes $(40 \%)$, and poor state of the vaccine vial monitor $(33 \%)$.

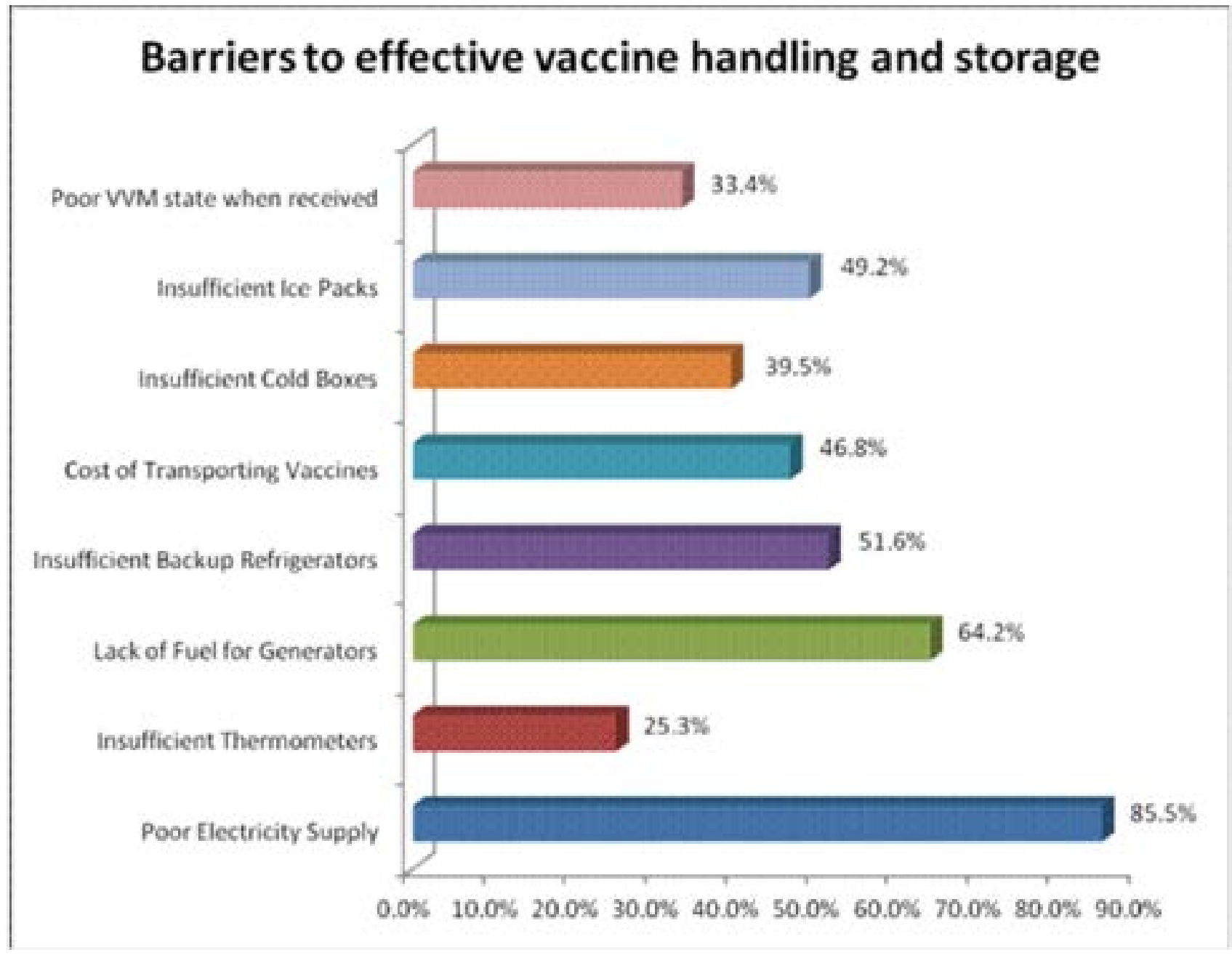

\section{Figure 1: Barriers to Effective Vaccine Handling and Storage as specified by respondents}

Association between awareness and use of vaccine handling and storage guidelines, training on vaccine management, knowledge of vaccine management and vaccine handling and storage practices.

Respondents $(70 \%)$ were aware of a vaccine handling and storage guideline, and had good vaccine handling and storage $(\mathrm{p}=0.004)$. Among those who were aware of a vaccine handling and storage guideline and had ever read such guideline, $73 \%$ had good practices $(p<0.001)$. Respondents $(61 \%)$ had received formal training on vaccine handling and storage and had good practice $(p=0.005)$. Ninety percent of respondents with good knowledge had good practices. 
Table 2: Association between Awareness, Use of Vaccine Handling and Storage Guideline, Training on Vaccine Management, Knowledge of Vaccine Management, and Vaccine Handling and Storage Practices

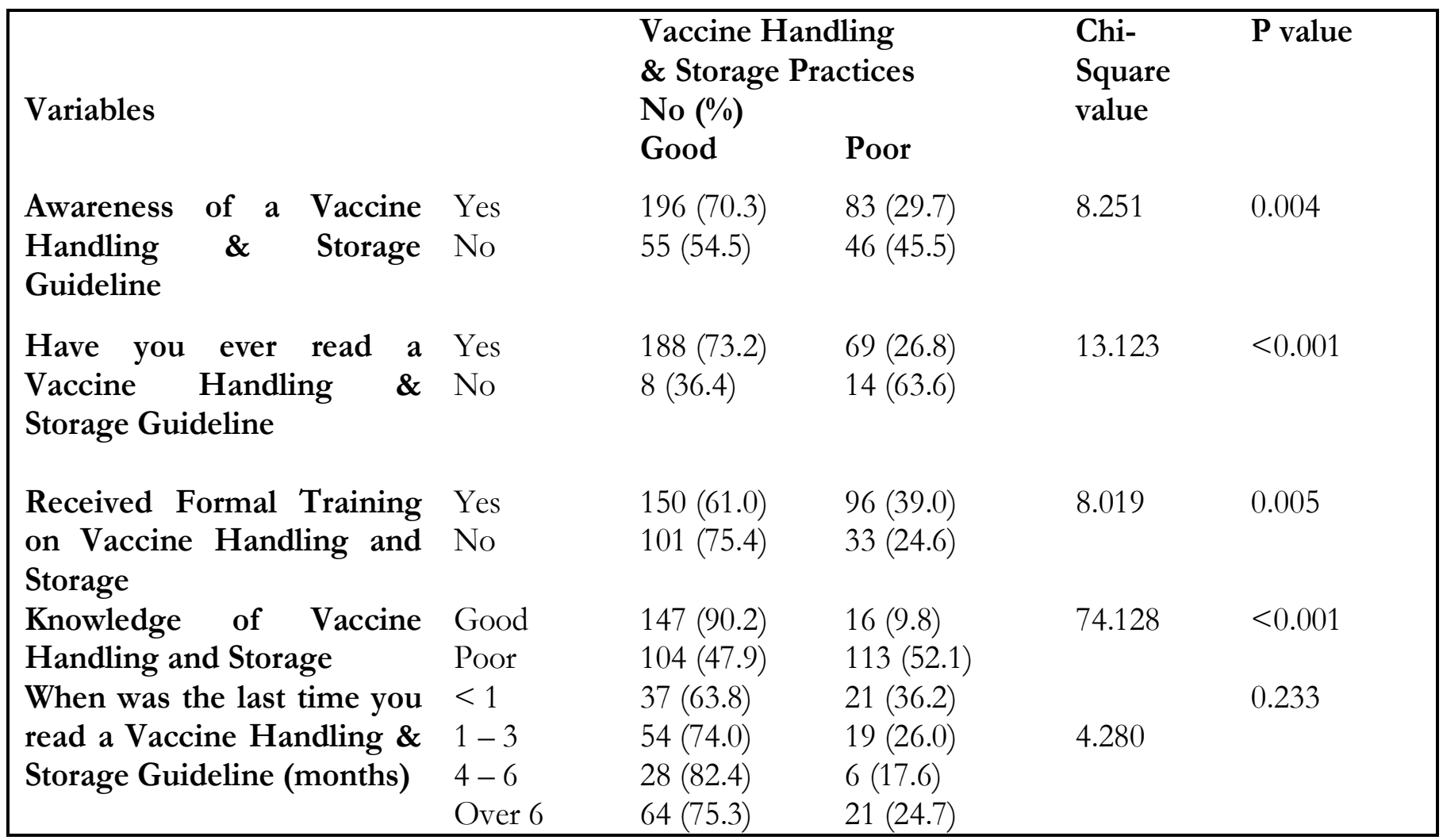

Predictors of respondents' vaccine handling and storage practices

After logistic regression analysis, utilization of a vaccine handling and storage guideline ( $\mathrm{OR}=2.490$, $95 \% \mathrm{CI}=1.588-3.906, \mathrm{P}=0.001$ ), exposure to formal train- ing on vaccine management $(\mathrm{OR}=5.280,95 \% \mathrm{CI}=2.502$ $-11.142, \mathrm{P}=0.001)$, and level of knowledge of vaccine handling and storage $(\mathrm{OR}=9.995,95 \% \mathrm{CI}=5.278-18.939$, $\mathrm{P}=0.001)$ were found to be predictors of vaccine handling and storage practices. Results are presented in Table 3. 
Table 3: Predictors of Respondents' Vaccine Handling and Storage Practices

\begin{tabular}{|c|c|c|c|}
\hline $\begin{array}{l}\text { Variables } \\
\text { Awareness of Vaccine Handling and Storage } \\
\text { Guideline }\end{array}$ & Odds Ratio & 95\% C.I & P Value \\
\hline Yes & 0.331 & $0.085-1.290$ & 0.111 \\
\hline $\begin{array}{l}\text { No (ref) } \\
\text { Have you read a Vaccine Handling and } \\
\text { Storage Guideline }\end{array}$ & 1 & & \\
\hline $\begin{array}{l}\text { Yes } \\
\text { No (ref) }\end{array}$ & $\begin{array}{l}2.490 \\
1\end{array}$ & $1.588-3.906$ & $<0.001$ \\
\hline $\begin{array}{l}\text { Received Formal Training on Vaccine } \\
\text { Handling and Storage }\end{array}$ & & & \\
\hline $\begin{array}{l}\text { Yes } \\
\text { No (ref) }\end{array}$ & $\begin{array}{l}5.280 \\
1\end{array}$ & $2.502-11.142$ & $<0.001$ \\
\hline $\begin{array}{l}\text { Knowledge of Vaccine Handling and Storage } \\
\text { Good } \\
\text { Poor(ref) }\end{array}$ & $\begin{array}{l}9.995 \\
1\end{array}$ & $5.275-18.939$ & $<0.001$ \\
\hline
\end{tabular}

\section{Spot observation}

Continuous temperature loggers that track and record refrigerator and freezer temperature were not attached to any of the units at the local storage sites.

Temperature-sensitive alarms that alert the cold chain officer via SMS during prolonged exposure to out-of-range temperatures were also not available at the storage sites

\section{Discussion}

Delayed and inadequate handling have been reported to be the main cause of loss of vaccine potency in Nigeria, not expiration or time to expiration ${ }^{1,8}$. This calls for utmost attention to vaccine handling and storage as it is essential in ensuring vaccine potency and maximizing immunisation efficacy. Several studies have made this suggestion $^{1,6,8,9,10}$.

Though a large proportion of respondents had received formal training on vaccine handling and storage, there still existed knowledge and practice gaps. This is consistent with a study in another developing country ${ }^{5}$. Factors associated with respondents' vaccine handling and storage practices $(p=0.05)$ include awareness of a vaccine handling and storage guideline, utilization of such guideline, exposure to formal training on vaccine management, and knowledge of vaccine handling and storage. Predictors of vaccine handling and storage practices were found to be utilization of vaccine handling and storage guideline, exposure to formal training on vaccine management, and level of knowledge of vaccine handling and storage. Awareness of vaccine handling and storage guideline was not a significant predictor of vaccine handling and storage practices ${ }^{11}$.

This study brings to the forefront several challenges to effective cold chain management such as highly unstable power supply as also reported by other studies on cold chain management in developing countries ${ }^{1,3}$. The infrequent and highly unreliable power supply in the region inhibits attaining recommended storage temperatures, making the experience of out-of-range temperatures (too hot) almost a formality. Coupled with lack of fuel for standby generators, this greatly accelerates vaccine thawing which in turns accelerates the loss of potency. Insufficient cold chain equipments at any level of the cold chain particularly the LGA level which is characterized by considerable decrease in vaccine potency ${ }^{1}$ results in poor vaccine handling and storage. This accelerates the progression of loss of vaccine potency and may lead to administration of sub-potent vaccines to clients who end up being at risk of contracting the diseases even after vaccination. 
Lack of continous temperature loggers makes it impossible to track vaccine temperatures when the cold chain officer is absent or during non-working days and hours. Any exposure to out-of-range temperature during these periods will remain undetected, as the temperature may have returned to normal when the stores reopen. Frozen vaccines usually develop hairline fractures during defrosting $^{12}$. Contamination of such vaccine vials may occur due to bacterial entry through the cracks. Provision and installation of temperature sensitive alarms will play a huge role in informing and initiating prompt response to temperature excursions.

\section{Limitation of study}

Documentation on vaccines stored outside recommended temperature range does not allow for inference about the potency of the vaccines. Testing vaccine vials to ascertain potency when found to be improperly stored is expensive and beyond the scope of this study.

In addition, study results portray a snapshot of the level of knowledge of vaccine handling and storage and its associated practices. There was however, no revisit to ascertain any change in knowledge and practice level of respondents.

\section{Conclusion and recommendation}

The basis for any cold chain management activity is ensuring potent vaccines get to and are administered to clients. Without capable personnel to properly manage cold chain equipments guided by stipulated procedures, all cold chain activities end in futility as sub-potent vaccines end up being administered to clients. This only becomes evident when vaccinated individuals contract with the disease the vaccines were meant to prevent, and is usually not attributed to poor handling of vaccines. Since vaccines are relatively very fragile biological agents, addressing the knowledge and practice gaps of vaccine handlers cannot be over-emphasized. With results from this study showing a strong association between level of knowledge of vaccine management and vaccine management practices, the need arises to constantly, update knowledge of vaccine handlers about vaccine management through trainings and frequent monitoring and supervision. This has been suggested by numerous studies on vaccine and cold chain management ${ }^{1,3,5,6,10}$. The National Primary Health Care Development Agency (NPHCDA) should ensure the National Immunisation Policy document is available and utilized in all cold stores and health facilities conducting RI.

To address the issue of prolonged power outages, generators (staffed or unmanned) and/or solar- powered inverters need to be in place. For efficient temperature logging and monitoring, continuous temperature-logging devices and temperature-sensitive alarms should be installed.

\section{Conflict of interest}

We declare no conflict of interest in the course of this study. The cost of funding this research was borne by the researchers.

\section{Acknowledgments}

We thank all the staff of the respective local government who responded to our questionnaires.

\section{References}

1. Oyefolu AOB, Nwaeke AC, Audu RA, Akinyemi KO, Salu OB, Muller CP, Omilabu SA. Evaluation of Measles Vaccine Cold Chain in Lagos State, Nigeria. African Journal of Clinical and Experimental Microbiology 2007; 8(1): 1-7 2. Ladhani S, Heath PT, Slack MP, McIntyre PB, DiezDomingo J, Campos J, Dagan R, Ramsay ME. Haemophilus influenza serotype $b$ conjugate vaccine failure in twelve countries with established national childhood immunisation programmes. Clinical Microbiology and Infection 2010; 16 (7)

3. Berhane Y, Demissie M. Cold Chain Status at Immunisation Centres in Ethiopia. East African Medical Journal 2000; 77(9): 476-479

4. Parmar D, Baruwa EM, Zuber P, Kone S. Impact of wastage on single and multi-dose vaccine vials: Implications for introducing pneumococcal vaccines in developing countries. Human Vaccines 2010; 6(3): 270-278

5. Joao Carlos de TM, Gunnar B. Cold Chain Management: Knowledge and practice. Ethiopian Journal of Health Development 2007; 21(2): 130-135

6. Bankole AM, Olusegun KK, Marian NB, Iboma G, Adebowale AO, Lukeman AJS, Olufemi O, Adetokunbo T, Odunaiye AM. The Impact of Health Facility Monitoring on Cold Chain Management Practices in Lagos, Nigeria. Journal of Public Health and Epidemiology 2010; 2(4): 78-81

7. NPC (2006) National Population Commission, Federal Government of Nigeria Abuja.

8. Baba MM, Omede SC, Omotara BA, Ambe JP. Evalu- 
ation of Measles Vaccines in North-Eastern Nigeria. $\mathrm{Na}$ ture and Science 2007; 5(3): 52 PubMed

9. Larry KP, Gregory W, Lance R. Too Hot, Too Cold: Issues with Vaccine Storage. Pediatrics 2006;118:1738-1739 10. Rao S, Naftar S, Baliga S, Unnikrishnana B. Evaluation, Awareness, Practice and Management of Cold Chain at the Primary Health Care Centers in Coastal India. Journal of Nepal Paediatric Society 2012; 32(1): 19-22 11. Bell KN, Hogue CJ, Manning C, Kendat A. Risk Factors for Improper Vaccine Storage and Handling in Private Provider Offices. Paediatrics 2001; 107(6): 1-6 12. Gazmararian JA, Oster NV, Green DC, Schuessler L, Howell K, Davis J, Krovisky M, Warburton SW. Vaccine Storage Practices in Primary Care Physician Offices: As- sessment and Intervention. American Journal of Preventive Medicine 2002; 23(4):

13. Matthias DM, Robertson J, Garrison MM, Newland S, Nelson C. Freezing Temperature in the Vaccine Cold Chain: A Systematic Literature Review. Vaccine 2007; 25: 3980-3986

14. Oladokun RE, Lawoyin TO, Adedokun BO: Immunization status and its determinants among children of female traders in Ibadan, South-Western Nigeria. Afr J Med Med Sci. 2009 Mar;38(1):9-15.

15. Brown VB, Oluwatosin OA.Socio-demographic factors associated with childhood immunization uptake in Akinyele Local Government Area, Oyo State, Nigeria. Afr J Med Med Sci. 2012 Jun;41(2):161-7. 